\title{
CAMBOYA 2018: DESAPARECIENDO LAS DEMOCRACIAS
}

\author{
John Marston \\ El Colegio de México
}

\section{TRASFONDO POLÍTICO}

La democracia liberal multipartidaria en Camboya quizá siempre tuvo el aspecto de un teatro que legitimaba otras relaciones de poder más patrimoniales, las cuales se encuentran justo debajo de la superficie. El primer ministro Hun Sen, del Partido Popular Camboyano (PPC), se había mostrado vulnerable durante las elecciones nacionales previas de 2014, en las que el grupo opositor, el Partido Camboyano de Rescate Nacional (PCRN), recibió 44\% de los votos en el conteo oficial (Parkhouse, 2018a), y aún más si se tienen en cuenta los comentarios de los protestantes. El resultado de las elecciones locales de junio de 2017 evidenció la fragilidad del PPC en el poder y su liderazgo político que perseguía estrategias de realpolitik para asegurar que no habría una oposición creíble para el partido en las elecciones nacionales de 2018. Uno de los dos fundadores del PCRN, Sam Rainsy, había estado exiliado en Francia desde 2016, enfrentando un arresto por difamación, en febrero de 2017 renunció al liderazgo del partido, previo a las elecciones comunales. En septiembre de 2017, el líder que aún permanecía activo, Khem Sokha, fue arrestado con los cargos de querer realizar una violenta "revolución de colores" en el país, y de traición en el juicio que se realizó en un solo día (McCargo, 2018). En noviembre del mismo año, el gobierno ordenó la disolución del PCRN, la mayoría del liderazgo central, quienes, incluidos los MP del PCRN, se vieron obligados a abandonar el país. A 118 líderes del partido se les prohibió participar en actividades políticas por un periodo de cinco años, así que algunos de nivel local del PCRN se sintieron obligados a unirse al PPC. 
Lo anterior corresponde a una supresión general, de mayor alcance, iniciada a mediados de 2017, poco después de las elecciones comunales, sobre organizaciones no gubernamentales y medios de comunicación que fueron percibidos como hostiles al gobierno, algunos de ellos vínculados con Estados Unidos. Varios analistas vieron el inicio de la supresión desde tiempo atrás, con la implementación de las leyes relacionadas con las ONG y con partidos políticos, el deterioro de las relaciones con Estados Unidos y las crecientes políticas represivas del hijo de Hun Sen, Hun Manith, director de inteligencia militar. Algunos comentaristas también lo interpretaron como una evidencia de que Camboya se está alejando de occidente y acercando cada vez más a China. El incremento de la censura estatal en internet y en las redes sociales, quizás con la asistencia de China, pudo haber influido en la supresión de mediados de 2017, que se hizo particularmente evidente tiempo después. El Instituto Democrático Nacional, con base en Estados Unidos, una organización no gubernamental con apoyo financiero de USAID, cuya misión es promover el proceso de elecciones multipartido, fue cerrado en agosto de 2017. El gobierno camboyano eventualmente lo describe como una organización coludida con el esfuerzo de derrocar al gobierno camboyano. Más o menos al mismo tiempo, un cineasta australiano dedicado a hacer documentales, James Riketson, que viajó a Camboya regularmente durante varios años y que tenía fama de ser excéntrico y confrontativo, fue arrestado, aparentemente por volar un dron con una cámara sobre el rally del PCRN; esta acción fue interpretada después como un acto de espionaje. En septiembre, Hun Sen pidió a los cuerpos de paz de Estados Unidos que sus voluntarios abandonaran el país. En noviembre, después del cierre del PCRN, el gobierno investigó a la ONG de derechos humanos, el Centro Camboyano para los Derechos Humanos, por su posible cierre debido a "vínculos con el extranjero"; pudo influir en la decisión el hecho de que esta ONG fue fundada por Khem Sokha en 2002. Después de una serie de reclamos populares, esta ONG continuó con sus actividades.

Desde los tiempos de la misión de la Organización de Naciones Unidas (ONU) en Camboya (UNTAC), en 1992-1993, cuando la prensa privada apenas florecía en el país, el gobierno empezó a controlar fuertemente los medios de comunicación. Una excepción fueron las trasmisiones de Voice of America y Radio FreeAsia, muy populares (las dos tienen financiamiento de Estados Unidos para su programación; un alto porcentaje del contenido de los reportajes de Radio FreeAsia está en lengua camboyana). Desde la década de 1990, pequeñas estaciones de radio han retransmitido la programación de estos dos medios que 
argumentan que "no son políticos", aunque tienden a apoyar al sistema multipartido; además, no vacilan en hacer reportajes de crítica al PPC, en tanto cubren las actividades de los partidos de oposición. En agosto, todas las estaciones de radio de provincia que retrasmitían la programación de VOC y de RFA fueron cerradas, con el argumento de que debían impuestos (aunque todavía estaban disponibles en internet, radios de onda corta, y, en algunas partes de Camboya, mediante trasmisiones de amplitud modulada desde Tailandia). En noviembre, más o menos al mismo tiempo que fue disuelto el CPRN, dos exreporteros de RFA fueron arrestados, acusados de trabajar para la RFA y estar involucrados en espionaje.

En general, desde la década de 1990 Camboya ha permitido reportajes más críticos en la prensa escrita que en las trasmisiones por radio, especialmente en las publicaciones en inglés. Los dos periódicos en inglés más conocidos datan del periodo de la UNTAC, y son el Cambodia Daily y el Phnom Post. Ambos tienen una sólida reputación internacional y son vistos como campo de entrenamiento para periodistas, tanto camboyanos como internacionales (un tercer periódico en inglés, el Khemer Times, apareció en escena más tarde; a menudo se pasa por alto en las descripciones del periodismo camboyano, y es menos dado a cubrir asuntos controvertidos). El Cambodia Daily y el Phnom Post fueron fundados por estadounidenses y empleaban periodistas estadounidenses y occidentales, así como camboyanos, aunque de ninguna manera eran financiados por el gobierno de Estados Unidos como el VOA y el RFA; durante diez años, el Phnom Post fue propiedad de australianos. Probablemente muy pocos observadores internacionales pudieron detectar algún sesgo mayor en sus informes que el encontrado, por ejemplo, en los informes de la BBC o de los servicios de cable internacional.

En agosto de 2017, al Cambodia Daily le fue presentado un pago por impuestos al gobierno por la cantidad equivalente a 6.3 millones de dólares (Ball, 2018), y se le informó que la renovación de su licencia dependía del pago de esa cantidad en el plazo de un mes. El diario, que argumentaba estar operando con pérdidas, tomó esta medida como una manera de obligarlo a cerrar, e inmediatamente suspendió la publicación impresa. Su último número coincidió con el arresto de Khem Sokha e incluyó un reportaje del mismo, que en el encabezado decía: "Descenso a la dictadura absoluta". A su socio, el yerno de su fundador Bernard Krishner, le fue prohibido salir del país. El cierre del periódico fue reportado y denunciado extensivamente en la prensa internacional. El Cambodia Daily decidió seguir publicando internacionalmente y en internet, con su base fuera de Cambodia, aunque sólo 
mediante artículos de otras agencias de noticias que incluían a VOA. Una demanda por difamación contra el subdirector del Cambodia Daily, Deborah Krishner-Steele, continuó hasta 2018.

\section{ELECCIONES DE 2018}

Todo lo anterior abrió el escenario para 2018: “The Leadup to the July Elections”. En marzo, el Khmer Post, que desde 2008 era dirigido por el australiano Bill Clough, reveló que por varios meses había tratado de pagar la enorme cuenta por concepto de impuestos, que ascendía a 3.9 millones de dólares, pero, afirmaba, no constituía "algo político", y negaba que hubiera amenazas contra el periódico. Durante todo el proceso, el periódico intentó distanciarse del tono que rodeó el cierre del Cambodia Daily y de mantener una posición no confrontacional. No obstante, en mayo se anunció que el Phnom Post sería vendido a una firma de Malasia con vínculos con el gobierno de Hu Sen, y que el problema de impuestos del periódico sería resuelto al concretarse la transacción. Finalmente ocurrió un enfrentamiento con el personal directivo editorial cuando el nuevo publicista censuró el reportaje que hizo el periódico sobre la venta del mismo; esta situación hizo que varios integrantes del senior staff abandonaran el periódico (BBC, 2018). La prensa internacional y las organizaciones de derechos humanos mostraron esto como evidencia, lo mismo que el cierre del Cambodia Daily de las restricciones políticas a la libertad de prensa. El Phnom Post se mostró políticamente más cauteloso, pues, a diferencia del Cambodia Daily, continuó publicando en Cambodia, con una crítica más aguda, al menos que el Khmer Times.

Aunque con menos relación directa con las elecciones de 2018, los observadores de derechos humanos también mostraron su preocupación por la aprobación de la ley de lese majesté, modelada como las de Tailandia, que en ocasiones se habían usado para la supresión política. En mayo, un maestro de cincuenta años fue arrestado por escribir un mensaje en Facebook que las autoridades dijeron que acusaba a la familia real de colusión para suprimir al CPRN. Poco después, un barbero de 70 años fue arrestado también por subir un mensaje en Facebook que fue considerado antimonárquico. No obstante, la importancia política de la nueva ley fue evidente cuando, en junio, el ministro de justicia ordenó a la corte municipal de Phnom Penh iniciar los procedimientos legales contra el exlíder del CNPR, Sam Rainsy, 
por escribir en su página de Facebook que la declaración del rey pidiendo a la población que votara, fue hecha "bajo presión" (Ben, 2018a).

En mayo, después de un periodo de registro de dos semanas, fue anunciado que veinte partidos participarían en las elecciones de julio. Ninguno de estos partidos representaba una amenaza seria para el PPC, y quizá la mayoría ya había llegado a acuerdos para representar alternativas inocuas que no amenazaran, de ninguna manera, al poder real. El partido más calificado era el Funcipec, el ganador de las elecciones auspiciadas por la ONU en 1993 y que desde entonces había perdido importancia. Uno de los pocos momentos de drama real durante la campaña sucedió en junio, cuando el príncipe Norodom Ranariddh, cabeza del partido, resultó gravemente herido luego de un accidente automovilístico durante la campaña, en el que perdió la vida su joven esposa (Barron, 2018). En otras circunstancias, un accidente de este tipo podía provocar rumores de juego sucio; incluso hubo consenso en el sentido de que Hun Sen no había sacado ventaja para debilitar a la oposición. Los camboyanos en Phnom Penh a quienes se les preguntó sobre el accidente, dijeron que era la retribución karmica de Ranariddh, por adúltero y por votar a favor de la disolución del PCNR. En general, los 19 partidos no fueron tomados en serio por la población en general; algunos de ellos se quejaron de que no tuvieron suficiente dinero para pagar anuncios televisivos. En julio, en una acción particularmente absurda, el Comité de Elección Nacional (CEN) anunció políticas que prohibían a los miembros de cualquier partido hacer críticas a la plataforma de otro partido (Soth, 2018).

Sin embargo, al igual que en elecciones anteriores, a pesar de las leyes que lo prohíben y de los anuncios en contra, se entendió que los empleados del gobierno, incluidos militares y policías, estaban obligados a hacer campaña en favor del CPP. En los últimos años, Hun Sen ha hecho uso extensivo de Facebook como plataforma para presentar sus propuestas y hacer sobresalir a su comunidad política. Durante la campaña de 2018, quizá de una manera novedosa, se esperaba que los empleados del gobierno publicaran fotos de ellos mismos haciendo campaña.

Los camboyanos que participaban en mítines de campaña del PPC recibían algún pago; en Phnom Penh les dieron el equivalente a 100 pesos por persona, así que, considerando que varios miembros de una familia podían recibir estos pagos, finalmente la cantidad total era significativa. Por ejemplo, una familia a la cual conozco, que durante varios años ha apoyado 
al PCRN, no tuvo ningún escrúpulo en seguir haciéndolo y asistió al rally por los 600 pesos que obtendría.

En mayo, las dos ONG mejor establecidas que han monitoreado las elecciones, Comfrel y NICFEC, anunciaron que no participarían como observadores en las elecciones de julio, argumentando los modos en que habían sido obstaculizadas sus actividades en las elecciones locales de 2017, lo mismo que el miedo a las repercusiones hacia los participantes voluntarios (Mech, 2018). Estados Unidos y la Unión Europea retiraron su apoyo financiero y el monitoreo de la elección que habían dado en elecciones previas (Allard y Park, 2018); Australia también decidió no mandar monitores, como lo hizo en junio la asociación internacional Transparencia Internacional. Japón, que originalmente señaló que mandaría monitores, al último minuto decidió no hacerlo. Pero China, Myanmar, Singapur y un número indeterminado de organizaciones internacionales sí enviaron monitores; se hizo una acusación de que algunas de estas organizaciones tenían la reputación de "ser observadores de elecciones contratados". De los observadores de las elecciones locales, la gran mayoría pertenecían a la Federación Juvenil Unida de Camboya (FJUC), cuyo director era uno de los hijos de Hu Sen, Hun Many, y a las Mujeres Camboyanas por la Paz y el Desarrollo (MCPD), dirigida por el viceprimer ministro, Men San An, igualmente prominente figura del PPC.

El PCNR, naturalmente, llamó al boicot de las elecciones. En abril, la Comisión Nacional de Elecciones (CNE) proclamó que los llamados a boicotear la elección eran actos criminales. Más tarde, Hun Sen llegó más lejos, al tachar los llamados a boicotear la elección como "traiciones". Como en muchos países, es costumbre en Camboya marcar con tinta el dedo del votante, como una forma de prevenir que voten más de una vez. Esto significa, no obstante, que un “dedo limpio", como es llamado por el PCNR, sería una identificación pública de que alguien no ha votado. Esto, especialmente en áreas rurales, en donde la interacción con funcionarios locales es generalmente una necesidad, puede tener repercusiones negativas, como se hizo evidente en algún momento. El Ministerio de Justicia también anunció que perseguiría a cualquiera cuyo dedo indicara que no había votado (Niem, 2018). Más tarde, en la campaña, el ministro del interior anunció que perseguiría a cualquiera que mostrara un dedo limpio en línea. Un representante sindical reportó que a los trabajadores se les daba tiempo libre para que regresaran a sus provincias de origen y pudieran votar, pero sólo se les daría la compensación por ese tiempo si al regresar mostraban su dedo entintado (Parkhouse, 2018b). 
En abril se reportó que 80000 elementos de las fuerzas de seguridad serían asignados para cuidar la elección. Unos días antes de la elección hubo muestras de armas y otro equipo militar en un gran estadio de Phnom Penh: el mensaje, que muchos percibieron fue que era lo que se esperaba si se daba un levantamiento al momento de llevarse a cabo las elecciones. Estados Unidos, después de la represión de 2017, advirtió a sus ciudadanos tener precauciones al visitar el país; otras embajadas previnieron a sus ciudadanos sobre viajar a este país en tiempo de elecciones. Los hoteles de Phnom Penh se quejaron porque visitantes internacionales cancelaron su viaje por estas razones. No obstante, al final, con la oposición efectivamente eliminada y el resultado de la elección mostrado como una conclusión sabida, no había bases reales para levantamientos y la elección no tuvo contratiempos.

El voto fue de $82 \%$, más alto que en las elecciones de 2014, indicación clara de que el llamado al boicot fue inefectivo, y una buena indicación de qué tan efectivo fue el aparato político del PPC para obtener el voto. Además de otorgarles a los trabajadores tiempo libre, les pagaron el transporte en autobús para ir a casa y votar. Un signo de insatisfacción fue el número de votos inválidos, más grande que nunca antes: $9 \%$ (4 veces más que en la elección previa) (Asian Network for Free Elections, 2018b). El PPC ganó con 77.4\% del voto, y capturó los escaños del legislativo. Internacionalmente la elección fue calificada como sin sentido; un vívido ejemplo es la siguiente declaración de un grupo observador:

La Red Asiática para Elecciones Libres (RAEL), después de una cuidadosa reflexión ha determinado que la conducta en las elecciones camboyanas de 2018 fue todo menos justa y libre. Habiendo sido observadores en más de 50 elecciones en las dos últimas décadas, RAEL cree que ésta estaba más desprovista de procesos políticos verdaderamente democráticos que casi cualquier otra elección que haya presenciado [Asia Network for Free Elections, 2018b].

La elección fue criticada en declaraciones de Estados Unidos, Australia, la Unión Europea. China, por su parte envió felicitaciones a Camboya por su elección "suave y exitosa". 


\section{SECUELAS DE LAS ELECCIONES}

Aunque no hubo protestas en Camboya después de la elección, sí hubo, por ejemplo, protestas de camboyanos en Estados Unidos pidiendo a ese gobierno no reconocer los resultados de la elección. Hasta la fecha no lo ha hecho, aunque Camboya continúa moviéndose diplomáticamente cerca de China y recibe de éste país grandes cantidades de ayuda, mayores a las de los países occidentales. El país es dependiente económicamente, en el sentido de que goza de un estatus favorable y especial en su comercio tanto con Estados Unidos como con la Unión Europea. Durante las elecciones, los comentaristas mencionaron que tanto Estados Unidos como la Unión Europea podían castigar al país removiendo el estatus comercial favorable; está por verse si esto sucede. En años recientes la táctica preferida de Estados Unidos, tanto con Camboya como con otros países, ha sido poner restricciones para la obtención de visas a miembros clave del gobierno. Ya en diciembre de 2017 Estados Unidos anunció que estaba poniendo restricciones de visa a miembros del gobierno involucrados en represión política ocasionada por las protestas en Camboya. Inmediatamente después de las elecciones en Camboya, Estados Unidos, al proclamar que la elección no fue justa ni libre, anunció que estaría expandiendo las restricciones para obtener visa (Meixler, 2018). Una resolución de la Unión Europea de septiembre podría ir potencialmente más allá: llama a la congelación de los bienes de los altos funcionarios camboyanos, lo mismo que a una revisión del estatus libre de tarifas con la Unión Europea (Men, 2018).

Las organizaciones de derechos humanos se congratularon por el hecho de que, en los meses siguientes a las elecciones, varios prisioneros políticos fueron liberados, muchos con el perdón real (Human Rights Watch, 2018), incluidos seguidores y activistas del PCNR arrestados desde las protestas de 2014, los dos reporteros de FreeAsia mencionados antes (liberados bajo fianza, aunque continúan los procedimientos legales en contra de ellos); Sourn Serey Ratha, fundador de un pequeño partido; Tep Vanny, activista de derechos de la tierra que fue destacado en un filme documental, y el comentador político Kim Sok. Después, de manera dramática, el 10 de septiembre el líder Khen Sokha fue liberado por razones de salud, y puesto en arresto domiciliario en su casa de Phnom Penh; quedó estipulado que no podría tener comunicación con otras figuras del PCNR ni con extranjeros. Los comentaristas hicieron notar que la liberación de los prisioneros fue diseñada para paliar los comentarios internacionales negativos de las elecciones, lo que es un hecho claro. Es también algo 
característico del estilo de Hun Sen alternar entre dureza y generosidad, un estilo patrimonial en el que el ejercicio del poder personal tiene más peso que el estado de derecho.

El caso del cineasta australiano James Ricketson tiene sus propias peculiaridades. Después de un año en prisión fue llevado a juicio, dos semanas después de las elecciones. Aunque en el mes de julio escribió una carta ofreciendo disculpas a Hun Sen, su estilo recalcitrante fue visto en su comentario a los reporteros cuando era trasladado de su vehículo a la sala del juzgado: "Ellos todavía no me dicen para qué país se suponía estaba espiando". En el juicio, su carrera como cineasta fue explicada a profundidad; el aclamado cineasta australiano Peter Weir testificó en su favor. Nada que un observador occidental pudiera interpretar como evidencia de espionaje fue presentado para su prosecución, aunque hubo algunos mensajes de correo electrónico que mostraban simpatía con el PCNR. A finales de agosto fue encontrado culpable y sentenciado a seis años de prisión. Después, el cineasta de izquierda John Pilger, quien había apoyado al gobierno camboyano desde el socialismo de los ochenta, habló en apoyo a Ricketson. Más tarde, el 21 de septiembre, cuando escribía este capítulo, se anunció que Rickelson recibiría el perdón real a solicitud de Hun Sen. Su hija fue citada, y se dijo que una petición en línea con más de 107000 firmas fue factor para que lo liberaran (Hunt, 2018). Uno no puede dejar de sentir que el propósito de la detención era demostrar que el gobierno camboyano, con el liderazgo muy personal de Hun Sen, podía detener a cualquiera de manera arbitraria, retenerlos durante un año y luego celebrar un juicio simulado para encontrarlo culpable: Una vez que se envió ese mensaje, el encarcelamiento real no fue tan importante.

Aún había muchos motivos por los que podían protestar las organizaciones de derechos humanos. Human Rights Watch señaló que todavía quedaban diez prisioneros políticos (Human Rights Watch, 2018). Aunque Kem Sokha, ahora en arresto domiciliario, estaba en mucho mejores condiciones que antes; la disolución de su partido y su permanente detención todavía representaban un ataque arbitrario a la democracia multipartido. Algunos de los liberados, que permanecieron críticos al gobierno, eran claramente vulnerables al re-arresto. Kim Sok, en particular, dos semanas después de su liberación fue llamado a la corte, donde enfrentó, entre otras cosas, una multa por 200000 dólares, que todavía estaba obligado a pagar. En septiembre aún permanecía escondido y buscando asilo fuera de Camboya (Ben, 2018b). 
El otro evento significativo posterior a las elecciones fue el nombramiento del hijo mayor de Hun Sen, Hun Manet, como comandante de las fuerzas armadas, lo que lo convirtió en la segunda figura más importante en la milicia camboyana. En los últimos años, los hijos adultos de Hun Sen han asumido papeles más significativos en el gobierno. Hun Manet tiene un BA de la Academia Militar de West Point en Estados Unidos, un MA de la New York University y un Ph.D de la Bristol University, títulos que lo colocan como el posible sucesor de su padre. Fue promovido varias veces en 2018 y se convirtió en general de cuatro estrellas inmediatamente antes de las elecciones, quizá como un indicador de la importancia de asegurar el control absoluto y la lealtad de los militares al momento de la elección.

\section{ECONOMÍA, TRABAJO Y CONEXIONES GLOBALES}

Aunque resultó difícil (a finales de 2018) aislar el tópico de la economía durante el año anterior, fueron evidentes ciertas tendencias básicas que parece ser continuarán en el futuro inmediato. En cierto grado, Hun Sen está en lo correcto al sostener el crecimiento económico bajo su liderazgo, el cuál se espera que en 2018 sea de $6.9 \%$ que refleje un modelo de crecimiento consistente a lo largo de los últimos diez años, y haga de Camboya uno de los seis países del mundo con mayor crecimiento económico (Reuters, 2018). Este nivel de crecimiento es en parte una señal de lo débil que era la economía después de la devastación del periodo de Pol Pot y de una década de sanciones. Puede decirse que este crecimiento, en parte, representa un proceso de normalización más que un florecimiento de riqueza. Camboya, considerada por largo tiempo como uno de los países más pobres del mundo, ahora es considerada un país de ingresos medios bajos (se ha dicho que Hu Sen tiene la esperanza de convertir al país en uno de ingresos medios altos para el 2030, y en una nación desarrollada para 2050). No obstante la evidencia de progreso, no siempre es fácil medir el peso del crecimiento del Producto Interno Bruto (PIB) de Camboya en relación con su continua dependencia de la ayuda extranjera (que decrecerá con sus estatus de país de ingresos medios) y su deuda externa. Efectivamente, para un observador externo, hay una evidencia superficial del crecimiento urbano de Phnom Penh, con grandes proyectos de infraestructura y rascacielos que se incrementan si se les compara con los edificios que hay en otros países del sudeste asiático. Una cadena de centros comerciales de lujo de origen japonés, Aeon, abrió su segundo centro en Phnom Penh en 2018, algo que fue visto por muchos camboyanos como 
un signo de que el país se ha elevado a niveles de mundo moderno orientado hacia el consumo. También hay evidencia de edifícios privados y de desarrollo de infraestructura en la Camboya rural.

El crecimiento del PIB de Camboya está siendo impulsado por el turismo y las exportaciones de vestidos y agricultura. Las exportaciones de vestidos y de artículos deportivos hacia la Unión Europea y a Estados Unidos, sus dos mercados más importantes, en la primera mitad de 2018 se incrementó más de $10 \%$ en relación con el año anterior, no obstante las tensiones políticas (May, 2018). El turismo subió 12.7\% en el mismo periodo (no obstante la caída en turismo en algunos sectores por miedo a las protestas electorales), lo cual fue incentivado por turistas procedentes de China (tres veces mayor que los provenientes de Vietnam, seguido de otros países asiáticos) (Fresh News, 2018). La exportación de arroz bajó $10 \%$ en relación con el mismo periodo del año anterior (Cheng, 2018), probablemente por las fluctuaciones en el mercado. La evidencia es contradictoria en la medida en que el crecimiento del PIB se filtra hacia los camboyanos en el extremo inferior del espectro económico.

The Cambodian Development Review, en sus reportes regulares y sobre el desarrollo económico camboyano, incluye reportes sobre la población vulnerable. Aunque el más reciente reporte disponible es de finales de 2017, puede reflejar la situación que prevalece en 2018. Su reporte está

[...] basado en una encuesta de 360 trabajadores vulnerables y 120 trabajadores de la confección en noviembre de 2017. Las ganancias diarias reales promedio de los trabajadores más vulnerables aumentan año tras año, pero las de conductores de ciclo, pequeños vendedores de vegetales, carroñeros y trabajadores de campos de arroz, disminuyeron [Camboya Development Review, 2017].

Esto es consistente con reportes de noticias y con la evidencia anecdótica que señala, en general, una creciente brecha entre pobres y ricos en el país, con la pobreza entre la población agrícola, y especialmente acentuada en la mayoría de la población. Los trabajadores de fábricas, aunque todavía reciben sueldos marginales, han mostrado algún progreso en términos de la economía de efectivo. Esta tendencia es apoyada por el reporte de la UNDP de 2018 (a su vez basado en estudios hechos en 2014-2015), el cual enfatiza lo 
extenso de la pobreza en Camboya y muestra que $40 \%$ de la población rural experimenta “pobreza multidimensional”, en oposición al 7\% de la población urbana (Voun, 2018).

Factores tanto comerciales como políticos han impulsado el incremento de sueldos regulares para los trabajadores urbanos. En octubre de 2017, el sueldo mínimo para los trabajadores del vestido y artículos deportivos fue elevado $11 \%$, a 170 dólares al mes, efectivo a partir del 1 de enero de 2018 (Reuters, 2017), todavía bajo si se tiene en cuenta el incremento en los costos. Los incrementos en los sueldos son negociados por una comisión tripartita: gobierno, sindicatos de trabajadores y empleadores. Futuros incrementos también son discutidos, aunque algunos representantes sindicales remarcan que las consideraciones políticas representaron un factor especial al anunciarlas en un año de elección. Este sector ahora tiene 700000 trabajadores, con un alto porcentaje de mujeres.

La monetización de la economía y el auge de las microfinanzas han ayudado a algunos camboyanos del sector rural, aunque han dado como resultado un incremento en los niveles de deuda. La encuesta hecha por la Cambodian Development Review, por ejemplo, encontró a finales de 2017, que 55\% de los entrevistados, cultivadores de arroz, tenían deudas con un porcentaje de interés promedio de 2.2\% al mes (Cambodian Development Review, 2017: 18). Un reportaje de 2018 indica que la deuda de los hogares se había incrementado rápidamente en Camboya en los últimos 20 años, y que era de 2.9 mil millones de dólares en abril de ese año, arriba de los 2.35 del año previo (Hutt, 2018).

La creciente integración de las áreas rurales a la economía monetizada ha afectado el desempeño de parte de la población agrícola tradicional, y enfrentado la pérdida de su tierra por deudas (lo que reflejó, al menos en parte, la presión de la creciente economía orientada al mercado), fluctuaciones en los precios de las cosechas internacionales, vulnerabilidad ante la nueva ley de la tierra e invasión de jugadores, tanto extranjeros como camboyanos, bien posicionados, consolidando a su vez el control sobre grandes extensiones de tierra agrícola y forestal. Estos factores están empujando a la población rural hacia trabajo estacional en áreas urbanas de Camboya, para trabajar en fábricas o en la construcción, así como en plantaciones cercanas a la frontera con Tailandia. La migración laboral hacia Tailandia, de creciente importancia para la economía de Camboya, generalmente involucra la entrada ilegal y puede representar una variedad en las condiciones de trabajo, incluyendo el trabajo difícil en barcos de pesca, algo considerado como esclavitud. Un reporte de mayo de 2018, cuando se 
impulsaba el registro de trabajadores indocumentados camboyanos en Tailandia, mostraba que había 80000 no registrados y 320000 registrados, aunque algunos otros decían que el número era mucho más alto (Sen, 2018). Por ejemplo, un reporte de 2016 encontró que 60\% de los camboyanos que regresaban de trabajar en Tailandia decían que la motivación para irse a trabajar fuera de Camboya había sido su endeudamiento (Hutt, 2018). Los camboyanos más afortunados aprobaban exámenes que les permitían ir a Corea del Sur como trabajadores invitados; esto me fue dicho por un líder comunal en la parte rural de la provincia de Kampong Cham, que podía ganar en un mes lo que ganaba en Camboya en un año. En diciembre de 2017 la cuota de trabajadores camboyanos en Corea fue de 53 000, y se espera que suba (Mom, 2017).

Si se ve la situación financiera de Camboya de manera más amplia, podrá apreciarse que existe un debate acerca del nivel de la deuda pública internacional y el riesgo que implica. La deuda pública internacional de Camboya se calculaba en más de 6 mil millones de dólares para finales de 2017 (Moeun, 2017). De acuerdo con un análisis del Fondo Monetario Internacional, esta cantidad aún representa el 32\% del PIB, que pone al país en bajo riesgo por "deuda angustiada" (International Monetary Fund, 2017). No obstante, la vulnerabilidad de Camboya como resultado de su deuda externa se comenta cada vez con más frecuencia en los medios periodísticos. La cuestión, en parte, dentro de un espectro geopolítico más amplio, es el hecho de que dos terceras partes de tal deuda son con China, en este tiempo en que la influencia china en Camboya se está incrementando. A Camboya a veces se le compara con Sri Lanka, donde la deuda llegó a estar controlada por una compañía estatal china. A inicios de 2018 se anunció un programa para reducir su deuda externa por medio de una emisión de bonos (Chea, 2018).

La deuda mencionada se refiere a los préstamos que el gobierno chino ha otorgado para realizar proyectos estatales camboyanos, como carreteras y puentes; sin embargo, también existe controversia acerca de la inversión privada china y la inmigración a Camboya. Muchos de los altos edificios en Phnom Penh fueron construidos con dinero chino, y al igual que los grandes complejos de vivienda, se hicieron pensando que serían comprados por chinos. De acuerdo con un reporte, para finales de 2018 habrá 22828 apartamentos de lujo, un enorme incremento respecto de los 8947 que había en 2017 (Mao y Meng, 2018), de los cuales un alto porcentaje pertenece a chinos provenientes del continente. Ahora bien, en ningún lugar es más evidente la presencia china que en Sihanoukville, la ciudad portuaria de Camboya que 
también es centro turístico. Nuevas historias se han centrado en el rápido proceso en el que Sihanoukville, antes un destino de turismo de mochila, se convirtió en un centro como Macao, de casinos que pertenecen a chinos y orientado hacia el turismo chino, a tal grado que ya no es un destino al que puedan acceder turistas camboyanos u occidentales. El turismo chino en Sihanoukville se duplicó entre 2016 y 2017. "Calificado como un gran desarrollo, el Casino Blue Bay y condominios se anuncia como 'uno de los proyectos icónicos de la iniciativa Una Franja Una Ruta"” (Fifield, 2018). Más allá del turismo, 104 de 121 negocios, en 4.4 millas cuadradas de la Zona Económica Especial de Sinanoukville, pertenecen a chinos, contra quienes existen acusaciones en Sinaoukville de estar involucrados en actividades criminales, como lavado de dinero, tráfico de personas y casinos ilegales (Hor y O'Byrne, 2018). Se dice además que la inversión china en casinos se está extendiendo a las áreas costeras de la provincia de Kampot; y que se han efectuado protestas por tal desarrollo en las áreas costeras de la provincia de Koh Kong, cerca de la frontera con Tailandia.

\section{MEDIO AMBIENTE}

Este medio no es el adecuado para discutir la amplia gama de asuntos relacionados con el ambiente que enfrenta Camboya; algunos de ellos son la creciente irregularidad de concesiones sobre clima, que provocan sequías e inundaciones que pueden relacionarse con el cambio climático. Aún más peculiar es el impacto ambiental de los grandes proyectos de infraestructura y de los crecientes proyectos a gran escala para agricultura o la extracción de recursos naturales relacionados con el "desarrollo" de Camboya, tal como se describe líneas arriba. Como se afirmó en este capítulo, los proyectos de desarrollo, lo mismo que la consolidación de tierra y otros recursos en manos de familias bien ubicadas, han resultado en el desplazamiento de población vulnerable y en un mayor número de protestas de la población afectada. Este fenómeno no debe ser visto sólo como protestas realizadas por ONG internacionales de medioambiente, cuya agenda es por lo regular diferente, pues las protestas de medioambiente y de población desplazada, generalmente, se dan en respuesta a asuntos que están relacionados, tales como la desforestación, el desarrollo de áreas costeras para el fomento al turismo (como ya se mencionó), grandes esquemas de irrigación en la planicie del lago de Tonle Sap, consolidación de tierra para plantaciones azucareras y de caucho, así como el impacto ambiental por la extracción de sal. 
Entre las protestas internacionales más comunes se encuentran las relacionadas con la construcción de presas. Camboya, de hecho, es vulnerable a los efectos de las presas sobre el río Mekong y sus tributarios en países ubicados río arriba de Camboya que afectan, por ejemplo, la pesca de subsistencia y la conexión tradicional entre la agricultura y los patrones de inundación. A finales de 2017, los pueblerinos, muchos de ellos pertenecientes a minorías étnicas, protestaron luego de ser desalojados de una área que sería inundada debido a la construcción de nueva presa Bajo Sesan II, planeada desde 2004. La presa, un proyecto conjunto de la compañía china Hidrolancang International Energy, la vietnamita EVN Internacional y el Grupo Real Camboyano, cerró sus compuertas a finales de septiembre de 2017 (Phak y Chen, 2017), pero las protestas continuaron hasta octubre, pues en noviembre se vieron obligados a evacuar, y en febrero de 2018 se inundó una enorme área. Las familias recibieron una casa nueva y la elección entre 6000 dólares o cinco hectáreas de tierra, que calificaron como inadecuada.

Pero el evento ambiental más catastrófico en la región se dio cuando una presa en construcción, en el sur de Laos, colapsó el 24 de julio. Las olas resultantes arrastraron los pueblos cercanos a la presa. Alrededor de 6000 personas en Laos tuvieron que ser reubicadas; hasta septiembre de 2018 se había confirmado que 40 personas habían muerto y otras 98 estaban desaparecidas. Dos días después de este colapso las aguas alcanzaron Camboya, donde las inundaciones llegaron a 11 metros de altura y hubo miles de desplazados; algunos reportajes señalaron que el número de evacuados en esta ciudad llegó a 25 000, aunque no hubo reportes de personas muertas o desaparecidas (Ellis-Petersen, 2018).

\section{REFERENCIAS CONSULTADAS}

Allard, T. y C. T. Prak (2018), "Election Monitoring Groups in Cambodia Headed by PM’s son", 20 de julio.

The Asian Network for Free Elections (2018a), "2018 Cambodia National Assembly Elections: Pre-Election Assessment Report”. Disponible en: https://anfrel.org/anfrel2018-cambodia-pre-election-assessment-report/ (consultado el 24 de agosto de 2018). 
The Asian Network for Free Elections (2018b), The 2018 Cambodian Elections: Nothing But a Charade, comunicado de prensa, 2 de agosto.

Ball, M. (2018), "When the Presses Stop", The Atlantic, enero y febrero.

Barron, L. (2018), "Cambodian Prince Norodom Ranariddh Seriously Injured in Car Accident that Killed his Wife", Time, 18 de junio.

BBC (2018), "Phnom Penh Post: Firing and Resignations After Sale of Cambodian Daily", 7 de mayo.

Ben, S. (2018a), "Justice Moves in On Rainsy: Court Told to Act against Former Opposition Leader for Insulting King", The Phnom Penh Post, 11 de junio.

Ben, S. (2018b), “'I Know Hun Sen will not Forgive Me’, Says Kim Sok”, The Phnom Penh Post, 20 de septiembre.

Cambodia Development Reivew (2017), "Economy Watch-Domestic Performance", Cambodia Development Review, vol. 21(4), pp. 17-18.

Chea, V. (2018), “Cambodia to Reduce Foreign Debt”, Khmer Times, 16 de enero.

Cheang, S. (2018), "Cambodian Election Body Warns against Poll Boycott Calls", Associated Press, 9 de abril.

Cheng, S. (2018), “Experts say Year Won't Pan Out Well for Rice Exports”, The Phnom Penh Post, 7 de agosto.

Ellis-Petersen, H. (2018), "Laos Dam Collapse sends Floods into Cambodia, Forcing Thousands to Flee", The Guardian, 26 de julio.

Fifield, A. (2018), "This Cambodian City is Turning into a Chinese Enclave, and not Everyone is Happy", Washington Post, 29 de marzo.

Fresh News (2018), "Over 3M International Tourists Visit Cambodia in First Half of 2018", Fresh News, 27 de agosto.

Hor, K. y B. O'Byrne (2018), "Chinese Embassy admits to Issues in Sihanoukville While Lauding Overall Impact of Investment", Phnom Penh Post, 8 de febrero. 
Hutt, David (2018), "To be Down, Out and In Debt in Cambodia", Asia Times, 14 de septiembre.

Human Rights Watch (2018), “Cambodia: Free All Political Prisoners”, 12 de septiembre. Recuperado de: https://www.hrw.org/news/2018/09/12/cambodia-free-all-politicalprisoners (consultado el 20 de septiembre de 2018).

Hunt, L. (2018), “Australian Filmmaker James Ricketson Granted Royal Pardon in Cambodia", The Age, 21 de septiembre.

International Monetary Fund (2017), "Cambodia: Staff Report for the 2017 Article IV Consultation-Debt Sustainability Analysis", 30 de agosto.

Mao, T. y S. Meng (2018), “One Party to Rule them All”, Bangkok Post, 3 de septiembre.

May, K. (2018), "Despite Election Uncertainty, Exports Achieve Solid Growth", Khmer Times, 1 de agosto.

McCargo, D. (2018), “The Trouble with Turnout at Cambodia's Election”, Asia Times, 31 de julio.

Mech, D. (2018), "Nicfec Opts not to Observe, Citing Fears of Volunteers, Election Watchdog will not Field Monitors", 17 de mayo.

Meixler, E. (2018), “The U.S. is Expanding Cambodia Visa Bans Over Last Month's 'Flawed' Election", Time, 16 de agosto.

Men, K. (2018), “EU Approves Resolution Targeting Cambodia’s Ruling Party”, vOA Khmer, 14 de septiembre.

Moeun Nhean (2018), "Cambodia's Foreign Debt Rose to \$6 Billion in 2017 as Economists Ask State to be Transparent", Phnom Penh Post, 22 de diciembre.

Mom, K. (2017), “Korea Worker Quota to Rise”, Khmer Times, 7 de diciembre.

Niem C. (2018), "Ministry to Punish Fraudsters Who Ink Finger without Voting", Phnom Penh Post, 31 de mayo, p. 4. 
Parkhouse, A. (2018a), "Cambodia Clampdown was Long in the Planning: Military Intelligence Unit Led by Prime Minister Hun Sen's Son Was Making Plans to Undercut the Political Opposition as Early as 2016", Asia Times, 5 de abril.

Parkhouse, A. (2018b), "Fear and Loathing Define Cambodia's Election”, Asia Times, 27 de julio.

Phak, S. y D. Chen (2017), "Sesan Dam Goes Online, While PM Dismisses Environmental Concerns", Phnom Penh Post, 26 de septiembre.

Reuters (2017), "Cambodia Hikes Minimum Wage for Textiles Workers by 11 pct from $2018 ", 5$ de octubre.

Reuters (2018), "Cambodia Economy to Grow 6.9 pct this Year, Driven by Exports-World Bank", Reuters, 19 de mayo.

Sen, D. (2018), "80 000 Cambodian Workers are Working Illegally in Thailand", Khmer Times, 22 de mayo.

Soth, K. (2018), "Parties Question NEC Policy”, The Phnom Penh Post, 11 de julio, pp. 1-2.

Voun, D. (2018), “UNDP Report Finds 35\% of Cambodians Still Mired in Poverty", Phnom Penh Post, 28 de septiembre. 